\title{
Reinhard Kardinal Marx \\ Grußwort zur Festschrift anlässlich des Jubiläums 25 Jahre Pflege an der KSH München
}

Vor 25 Jahren war die Katholische Stiftungshochschule München die erste Hochschule in Bayern, die die Pflege wissenschaftlich an einer Hochschule implementierte. Durch die Verschränkung von Management, Pädagogik und Wissenschaft in der Pflege und der Einführung neuer Lernformen und des wissenschaftsinduzierten Lernens wurde die KSH München zur Vorreiterin bei der Akademisierung der Pflege.

Mit einer Schwerpunktbildung in den Bereichen Gesundheits-, Bildungs- und soziale Berufe besetzt die Hochschule entscheidende Zukunftsthemen und kann sich mit diesen thematischen Akzenten klar innerhalb einer äußerst dynamischen Hochschullandschaft profilieren. Die aktuelle Etablierung eines Studiengangs Hebammenkunde an der Fakultät Gesundheit und Pflege ist ein deutliches Signal, dieses Thema weiterzuentwickeln.

Mit der Einführung des Fachbereichs/der Fakultät Pflege lieferte und liefert die Hochschule auch einen Beitrag zur Behebung des Fachkräftemangels in wesentlichen Bereichen. Zudem fördert sie die Interdisziplinarität in der Pflege, wodurch heute interprofessionelle Teams im Gesundheitssektor Standard geworden sind. Auch führte die Akademisierung und Professionalisierung der Pflege zu einer Aufwertung und höherer gesellschaftlicher Anerkennung dieses sog. klassischen „Frauenberufs“. Pflege wird nicht mehr als etwas Privates angesehen, das einer angeborenen Fürsorgehaltung von Frauen entspringt, sondern sie wird in ihrer ganzen Komplexität und als gesellschaftliche Herausforderung wahrgenommen.

Verschiedene Faktoren sind verantwortlich für diese Erfolgsgeschichte: Der wichtigste Faktor ist das exzellente wissenschaftliche Personal, das die Fakultät trägt. Inzwischen ist die KSH München gerade im Pflegebereich die Hochschule, die bayernweit die Förderung des wissenschaftlichen Nachwuchses maßgeblich betreibt. Hinzu kommen die beeindruckenden Bildungspartnerschaften der Hochschule mit Kliniken, Fach- und Berufsverbänden, Anstellungsträgern und Praxisstellen. Zudem gelingt es der Fakultät, den Professions- und Wissenschaftsdiskurs aktiv zu gestalten. Außerdem begeht sie neue Wege des Lehrens und Lernens. Sie leistete Pionierarbeit mit der Entwicklung ihrer Simulations- und Skillslabore, in denen die Studierenden in vorgegebenen Szenarien mit einer Simulationspuppe oder mit Simulationspatient(inn)en an praxisnahe berufliche Anforderungen der Pflege herangeführt werden. Und nicht zuletzt zeigt sich hier das Profil einer Katholischen Hochschule: Alles Handeln in der Pflege geschieht aus ethischer Verantwortung. Der Mensch steht mit seiner von Gott gegebenen Würde im Mittelpunkt und alles geschieht für ihn und mit ihm. 
Für dieses enorme Engagement bin ich sehr dankbar und spreche diesen Dank auch als Vorsitzender der Freisinger Bischofskonferenz und ebenso für den Stiftungsrat als Trägerin der Hochschule aus. Ich wünsche allen, die an der Fakultät Gesundheit und Pflege der KSH München arbeiten, lehren und lernen Gottes reichen Segen für ihr weiteres Wirken.

Ihr

Reinhard Kardinal Marx

Erzbischof von München und Freising

Vorsitzender der Freisinger Bischofskonferenz 